PROCEEDINGS OF THE

AMERICAN MATHEMATICAL SOCIETY

Volume 127, Number 10, Pages 2931-2941

S 0002-9939(99)05017-0

Article electronically published on April 23, 1999

\title{
QUADRATIC $q$-EXPONENTIALS AND CONNECTION COEFFICIENT PROBLEMS
}

\author{
MOURAD E. H. ISMAIL, MIZAN RAHMAN, AND DENNIS STANTON
}

(Communicated by Hal. L. Smith)

\begin{abstract}
We establish expansion formulas of $q$-exponential functions in terms of continuous $q$-ultraspherical polynomials, continuous $q$-Hermite polynomials and Askey-Wilson polynomials. The proofs are based on solving connection coefficient problems.
\end{abstract}

\section{INTRODUCTION}

The $q$-exponential function on a $q$-quadratic grid is

$$
\mathcal{E}_{q}(x ; a, b):=\sum_{n=0}^{\infty} \frac{\left(a q^{(1-n) / 2} e^{i \theta}, a q^{(1-n) / 2} e^{-i \theta} ; q\right)_{n}}{(q ; q)_{n}} q^{n^{2} / 4} b^{n},
$$

where $x=\cos \theta[\mathrm{Is}: \mathrm{Zh}],[\mathrm{At}: \mathrm{Su}]$. Ismail and Zhang [Is:Zh] gave a $q$-analogue of the expansion of the plane wave in spherical harmonics. Their formula is

$\mathcal{E}_{q}(x ;-i, b / 2)=\frac{(q ; q)_{\infty}(2 / b)^{\nu}}{\left(-b^{2} / 4 ; q^{2}\right)_{\infty}\left(q^{\nu} ; q\right)_{\infty}} \sum_{n=0}^{\infty} i^{n}\left(1-q^{n+\nu}\right) q^{n^{2} / 4} J_{\nu+n}^{(2)}(b ; q) C_{n}\left(x ; q^{\nu} \mid q\right)$,

where $J_{\nu+n}^{(2)}$ are $q$-Bessel functions [Is], [Ga:Ra] and $C_{n}(x ; \beta \mid q)$ are the continuous $q$-ultraspherical polynomials [As:Is], [Ga:Ra]. This formula has attracted some attention and two different proofs were given in [Fl:Vi] and [Is:Ra:Zh]. The proof by Floreanini and Vinet [Fl:Vi] is group theoretic and is of independent interest. Equation (1.2) was extended to continuous $q$-Jacobi polynomials in [Is:Ra:Zh], where the following expansion was established:

$$
\mathcal{E}_{q}(x ;-i, r)=\sum_{m=0}^{\infty} a_{m} p_{m}\left(x ; b, b q^{1 / 2},-c,-c q^{1 / 2}\right),
$$

Received by the editors December 29, 1997.

1991 Mathematics Subject Classification. Primary 33D45; Secondary 42C15.

Key words and phrases. Askey-Wilson polynomials, continuous $q$-ultraspherical polynomials, $q$-exponential functions, $q$-Bessel functions.

The first author was partially supported by NSF grant DMS-9625459, the second author was partially supported by NSERC grant A6197, and the third author was partially supported by NSF grant DMS-9400510. 
with the $a_{m}$ 's given by

$$
\begin{aligned}
a_{m}= & \frac{\left(b^{2} c^{2}, b^{2} q^{1 / 2} ; q\right)_{m}\left(i r q^{1 / 2} ; q\right)_{\infty}}{\left(q, b c q^{1 / 2}, b c ; q\right)_{m}(-i r ; q)_{\infty}}(i r / b)^{m} q^{m^{2} / 4} \\
& \times{ }_{2} \phi_{1}\left(\begin{array}{c}
c q^{m / 2+1 / 4},-b q^{m / 2+1 / 4} \\
b c q^{m+1 / 2}
\end{array} \mid q^{1 / 2}, i r\right) .
\end{aligned}
$$

The purpose of this paper is to extend (1.3) to an Askey-Wilson polynomial expansion (see (2.7)), and thereby also several special cases: (4.1), (4.4), (4.6). We also specialize (2.7) to find expansions of $q$-Bessel functions instead of the quadratic $q$-exponential function, see (3.2), (3.3). The fundamental technique is a connection coefficient result for Askey-Wilson polynomials (Theorem 1), which is established from the Nassrallah-Rahman integral [Ga:Ra, (6.3.9)]

It is known that expansions of the type treated in this paper are equivalent to inversions of certain lower triangular matrices [Fi:Is] and to Lagrange and $q$ Lagrange inversion [Ge:St]. In $\S 6$ we give the matrices and inverse relations lying behind the expansions established in this paper. We explain a positivity result for the connection coefficients for certain Askey-Wilson polynomials in $\S 5$.

We record here some the definitions and properties of the $q$-Bessel functions that will be required. The $q$-Bessel functions $J_{\nu}^{(1)}(x ; q)$ and $J_{\nu}^{(2)}(x ; q)$ are defined by

$$
\begin{aligned}
& J_{\nu}^{(1)}(x ; q)=\frac{\left(q^{\nu+1} ; q\right)_{\infty}}{(q ; q)_{\infty}} \sum_{n=0}^{\infty} \frac{(-1)^{n}(x / 2)^{\nu+2 n}}{\left(q, q^{\nu+1} ; q\right)_{n}}, \\
& J_{\nu}^{(2)}(x ; q)=\frac{\left(q^{\nu+1} ; q\right)_{\infty}}{(q ; q)_{\infty}} \sum_{n=0}^{\infty} \frac{(-1)^{n}(x / 2)^{\nu+2 n}}{\left(q, q^{\nu+1} ; q\right)_{n}} q^{n(\nu+n)} .
\end{aligned}
$$

They are related through

$$
J_{\nu}^{(2)}(x ; q)=\left(-x^{2} / 4 ; q\right)_{\infty} J_{\nu}^{(1)}(x ; q) .
$$

We will also need

$$
{ }_{2} \phi_{1}\left(-q^{\nu+1},-q^{\nu+2} ; q^{2 \nu+2} ; q^{2},-b^{2} / 4\right)=\frac{(q ; q)_{\infty}}{\left(q^{\nu+1} ; q\right)_{\infty}} \frac{(2 / b)^{\nu}}{\left(-b^{2} / 4 ; q^{2}\right)_{\infty}} J_{\nu}^{(2)}(b ; q),
$$

which follows from (1.23) of [Ra] (note a misprint) via [Ga:Ra, (III.4)].

\section{A BASIC EXPANSION FORMULA}

We prove Theorem 1, which is the fundamental theorem in this paper. It allows one to expand any function of $\left(\alpha e^{i \theta}, \alpha e^{-i \theta} ; q\right)_{n}$ in terms of Askey-Wilson polynomials which are defined by (see [As:Wi] or [Ga:Ra])

$$
p_{n}(\cos \theta ; a, b, c, d):=(a b, a c, a d ; q)_{n} a^{-n} r_{n}(\cos \theta ; a, b, c, d),
$$

where

$$
r_{n}(\cos \theta ; a, b, c, d):={ }_{4} \phi_{3}\left(\begin{array}{c|c}
q^{-n}, a b c d q^{n-1}, a e^{i \theta}, a e^{-i \theta} \\
a b, a c, a d
\end{array} \mid q, q\right) .
$$

The main difference between $p_{n}$ and $r_{n}$ is that $p_{n}$ is symmetric in all four parameters while $r_{n}$ is symmetric in $b, c, d$ but not in $a$. The basic expansion formula that we 
shall prove in this section is

$$
\left(\alpha e^{i \theta}, \alpha e^{-i \theta} ; q\right)_{n}=\sum_{j=0}^{n} A_{n, j} p_{j}(x ; a, b, c, d)
$$

Theorem 1. The coefficients $A_{n, j}$ in (2.1) are given by

$$
\begin{aligned}
A_{n, j}= & \frac{(q, \alpha d ; q)_{n}(\alpha / d ; q)_{n-j}(a b c d / q ; q)_{j}}{(q, \alpha d ; q)_{j}(q ; q)_{n-j}(a b c d / q ; q)_{2 j}} \\
& \quad \times(-\alpha)^{j} q^{j(j-1) / 2}{ }_{4} \phi_{3}\left(\begin{array}{c|c}
q^{j-n}, a d q^{j}, b d q^{j}, c d q^{j} & \\
\alpha d q^{j}, d q^{1-n+j} / \alpha, a b c d q^{2 j} & q, q) .
\end{array}\right) .
\end{aligned}
$$

Proof. From the orthogonality relation of the Askey-Wilson polynomials, (7.5.15)(7.5.17) in [Ga:Ra], we find that

$$
\begin{aligned}
& \kappa A_{n, j}= h_{j} \int_{-1}^{1} \frac{h\left(x ; 1,-1, q^{1 / 2},-q^{1 / 2}, \alpha\right)}{h\left(x ; a, b, c, d, \alpha q^{n}\right)} p_{j}(x ; a, b, c, d) \frac{d x}{\sqrt{1-x^{2}}} \\
&=(a b, a c, a d ; q)_{j} a^{-j} h_{j} \sum_{k=0}^{j} \frac{\left(q^{-j}, a b c d q^{j-1} ; q\right)_{k} q^{k}}{(q, a b, a c, a d ; q)_{k}} \\
& \quad \times \int_{-1}^{1} \frac{h\left(x ; 1,-1, q^{1 / 2},-q^{1 / 2}, \alpha\right)}{h\left(x ; a q^{k}, b, c, d, \alpha q^{n}\right)} \frac{d x}{\sqrt{1-x^{2}}}
\end{aligned}
$$

where

$$
h(\cos \theta ; \alpha):=\left(\alpha e^{i \theta}, \alpha e^{-i \theta} ; q\right)_{\infty}, \quad h\left(x ; a_{1}, \ldots, a_{n}\right):=\prod_{j=1}^{n} h\left(x ; a_{j}\right)
$$

the $h_{j}$ 's are

$$
h_{j}=\frac{1-a b c d q^{2 j-1}}{1-a b c d / q} \frac{(a b c d / q ; q)_{j}}{(q, a b, a c, a d, b c, b d, c d ; q)_{j}} .
$$

and $\kappa$ is

$$
\kappa:=\frac{2 \pi(a b c d ; q)_{\infty}}{(q, a b, a c, a d, b c, b d, c d ; q)_{\infty}} .
$$

This is valid if $\max (|a|,|b|,|c|,|d|)<1$. The integral in (2.3) can be evaluated by the Nassrallah-Rahman integral [Ga:Ra, (6.3.8)], and is equal to

$$
\begin{gathered}
\frac{2 \pi\left(\alpha b, \alpha c, \alpha d, a b c d q^{k}, \alpha b c d q^{n} ; q\right)_{\infty}}{\left(q, b c, b d, c d, a b q^{k}, a c q^{k}, a d q^{k}, \alpha b q^{n}, \alpha c q^{n}, \alpha d q^{n}, \alpha b c d ; q\right)_{\infty}} \\
\times{ }_{8} W_{7}\left(\alpha b c d / q ; b c, b d, c d, \alpha q^{-k} / a, q^{-n} ; q, a \alpha q^{n+k}\right) \\
=\kappa(\alpha d, \alpha / d ; q)_{n}(a b, a c ; q)_{k} \\
\quad \times \sum_{m=0}^{n} \frac{\left(q^{-n}, b d, c d ; q\right)_{m} q^{m}}{\left(q, \alpha d, d q^{1-n} / \alpha ; q\right)_{m}} \frac{(a d ; q)_{k+m}}{(a b c d ; q)_{k+m}},
\end{gathered}
$$


where (III.17) of [Ga:Ra] was used in the last step. Thus the $A_{n, j}$ 's have the representation

$$
\begin{aligned}
A_{n, j}=(a b, a c, a d ; q)_{j} a^{-j} h_{j}(\alpha d, \alpha / d ; q)_{n} & \sum_{m=0}^{n} \frac{\left(q^{-n}, a d, b d, c d ; q\right)_{m}}{\left(q, d \alpha, d q^{1-n} / \alpha, a b c d ; q\right)_{m}} q^{m} \\
& \times{ }_{3} \phi_{2}\left(\begin{array}{c|c}
q^{-j}, a b c d q^{j-1}, a d q^{m} & q, q) . \\
a b c d q^{m}, a d &
\end{array}\right)
\end{aligned}
$$

The ${ }_{3} \phi_{2}$ in (2.4) is now summed by (II.12) in [Ga:Ra]. After some simplification we establish (2.2), completing the proof of Theorem 1.

Application of the Sears transformation [Ga:Ra, (III.15)] gives an alternate representation of $A_{n, j}$, which we will find useful later in this work. It is

$$
\begin{aligned}
A_{n, j}= & \frac{(q, c \alpha, d \alpha, a b ; q)_{n}(a b c d / q ; q)_{j}}{(q, c \alpha, d \alpha, a b ; q)_{j}(q ; q)_{n-j}(a b c d ; q)_{n+j}} \frac{1-a b c d q^{2 j-1}}{1-a b c d / q}(-\alpha)^{j} q^{j(j-1) / 2} \\
& \times{ }_{4} \phi_{3}\left(\begin{array}{c|c}
q^{j-n}, c d q^{j}, \alpha / a, \alpha / b & \\
\alpha c q^{j}, \alpha d q^{j}, q^{1-n} / a b & q, q
\end{array}\right) .
\end{aligned}
$$

It is clear from (2.1) that $A_{n, j}$ must be symmetric in $a, b, c, d$ which, however, is not obvious in either of the two forms (2.2) or (2.5). The symmetry becomes explicit when we apply the Watson transformation [Ga:Ra, (III.17)] to obtain the following representation:

$$
\begin{aligned}
A_{n, j}= & \frac{(q, a \alpha, b \alpha, c \alpha, d \alpha ; q)_{n}\left(a b c d / q, \alpha^{2} ; q\right)_{j}}{(q, a \alpha, b \alpha, c \alpha, d \alpha ; q)_{j}\left(\alpha^{2} ; q\right)_{n}(q ; q)_{n-j}} \frac{1-a b c d q^{2 j-1}}{1-a b c d / q} \frac{(-\alpha)^{j} q^{j(j-1) / 2}}{(a b c d ; q)_{n+j}} \\
& \times{ }_{8} W_{7}\left(\alpha^{2} q^{j-1} ; q^{j-n}, \alpha / a, \alpha / b, \alpha / c, \alpha / d ; a b c d q^{n+j}\right)
\end{aligned}
$$

In the subsequent sections we shall use Theorem 1 in the following way. We have

$$
\sum_{n=0}^{\infty} c_{n}\left(\alpha e^{i \theta}, \alpha e^{-i \theta} ; q\right)_{n}=\sum_{j=0}^{\infty} p_{j}(\cos \theta ; a, b, c, d) \sum_{n=0}^{\infty} A_{n+j, j} c_{n+j},
$$

which is obtained from (2.1) by multiplying by $c_{n}$ and and summing over $n$, where $\left\{c_{n}\right\}$ is an arbitrary sequence, provided that the left-hand side of (2.7) converges and interchanging the sums is justifiable. If we choose $\alpha=e q^{(1-n) / 2}$ for some constant $e$ and $c_{n}$ in accordance with (1.1), then (2.7) is an expansion for a $q$-exponential function $\mathcal{E}_{q}$. We will specialize the parameters in such a way that the ${ }_{4} \phi_{3}$ series in (2.2) and (2.5), or the ${ }_{8} W_{7}$ series in (2.6), can be summed. We will also choose $\alpha$ independent of $n$, obtaining basic hypergeometric series expansions.

\section{EXPANSIONS IN CONTINUOUS $q$-ULTRASPHERICAL POLYNOMIALS}

In this section we first find two general Askey-Wilson polynomial expansions with $q$-Bessel functions, (3.2) and (3.3). First, if $\alpha=a$, then $A_{n, j}$ is summable by (2.5). Next we specialize to $\alpha=-i q^{(1-n) / 2}$ and the continuous $q$-ultraspherical polynomials, and prove (1.2).

If $\alpha=a$, and

$$
c_{n}=\frac{\left(E_{1}, E_{2} ; q\right)_{n}}{(q, a b, a c, a d ; q)_{n}}\left(-B^{2} / 4\right)^{n},
$$


then (2.5) and (2.7) imply

$$
\begin{aligned}
\sum_{n=0}^{\infty} & \frac{\left(a e^{i \theta}, a e^{-i \theta}, E_{1}, E_{2} ; q\right)_{n}}{(q, a b, a c, a d ; q)_{n}}\left(-B^{2} / 4\right)^{n} \\
= & \sum_{j=0}^{\infty} \frac{\left(a b c d / q, E_{1}, E_{2} ; q\right)_{j}}{(q, a b, a c, a d ; q)_{j}} \frac{1-a b c d q^{2 j-1}}{1-a b c d / q}\left(a B^{2} / 4\right)^{j} q^{j(j-1) / 2} p_{j}(x ; a, b, c, d) \\
& \times{ }_{2} \phi_{1}\left(\begin{array}{c|c}
E_{1} q^{j}, E_{2} q^{j} & q,-B^{2} / 4 \\
a b c d q^{2 j} &
\end{array}\right)
\end{aligned}
$$

Setting $a b c d=q^{2 \nu+1}, E_{1}=-q^{\nu+1 / 2}$, and $E_{2}=-q^{\nu+1}$, in (3.1) and using (1.6) we find

$$
\begin{aligned}
& { }_{4} \phi_{3}\left(\begin{array}{c|c}
a e^{i \theta}, a e^{-i \theta},-q^{\nu+1 / 2},-q^{\nu+1} & q,-\frac{B^{2}}{4} \\
a b, a c, a d &
\end{array}\right. \\
& =\frac{\left(q^{1 / 2} ; q^{1 / 2}\right)_{\infty}}{\left(q^{\nu+1 / 2} ; q^{1 / 2}\right)_{\infty}\left(-b^{2} / 4 ; q\right)_{\infty}} \sum_{j=0}^{\infty} \frac{\left(q^{2 \nu} ; q\right)_{j}\left(q^{\nu+1 / 2} ; q^{1 / 2}\right)_{2 j}}{(q, a b, a c, a d ; q)_{j}} \frac{1-q^{2 \nu+2 j}}{1-q^{2 \nu}} q^{j(j-1) / 2} a^{j} \\
& \times(2 / b)^{2 \nu} J_{2 \nu+2 j}^{(2)}\left(B ; q^{1 / 2}\right) p_{j}(x ; a, b, c, d) .
\end{aligned}
$$

We may find another $q$-Bessel expansion by choosing $E_{1}=E_{2}=0$ in (3.1), and using (1.4a) and (1.5). The result (again for $a b c d=q^{2 \nu+1}$ ) is

$$
\begin{aligned}
& { }_{4} \phi_{3}\left(\begin{array}{c|c}
a e^{i \theta}, a e^{-i \theta}, 0,0 & \left.q,-\frac{B^{2}}{4}\right) \\
a b, a c, a d &
\end{array}\right. \\
& =\frac{(q ; q)_{\infty}(2 / B)^{2 \nu}}{\left(q^{2 \nu+1},-B^{2} / 4 ; q\right)_{\infty}} \sum_{j=0}^{\infty} \frac{\left(q^{2 \nu} ; q\right)_{j}\left(q^{2 \nu+1} ; q\right)_{2 j}}{(q, a b, a c, a d ; q)_{j}} \frac{1-q^{2 \nu+2 j}}{1-q^{2 \nu}} q^{j(j-1) / 2} a^{j} \\
& \quad \times J_{2 \nu+2 j}^{(2)}(B ; q) p_{j}(x ; a, b, c, d) .
\end{aligned}
$$

The continuous $q$-ultraspherical polynomials $C_{n}\left(x ; a^{2} \mid q\right)$ are obtained from the Askey-Wilson polynomials by

$$
p_{n}(x ; a, a \sqrt{q},-a,-a \sqrt{q})=\frac{(q ; q)_{n}\left(a^{4} ; q\right)_{2 n}}{\left(a^{2}, a^{4} ; q\right)_{n}} C_{n}\left(x ; a^{2} \mid q\right) .
$$

Thus $q$-ultraspherical versions of (3.2) and (3.3) can be found be putting

$$
\{a, b, c, d\}=\left\{q^{\nu / 2}, q^{(\nu+1) / 2},-q^{\nu / 2},-q^{(\nu+1) / 2}\right\} .
$$

The left-hand sides then become other $q$-analogues of the Bessel function $J_{\nu+1 / 2}$.

Next we consider $(2.7)$ for the continuous $q$-ultraspherical polynomials $(b=$ $a \sqrt{q}, c=-a, d=-a \sqrt{q}$ ) for an arbitrary $\alpha$. Using (3.4.7) in [Ga:Ra], we have

$$
\begin{aligned}
& { }_{8} W_{7}\left(\alpha^{2} q^{j-1} ; q^{j-n}, \alpha q^{-1 / 2} / a,-\alpha q^{-1 / 2} / a, \alpha / a,-\alpha / a ; q, a^{4} q^{n+j+1}\right) \\
& =\frac{\left(\alpha^{2} q^{j}, a^{4} q^{2 j+1} ; q\right)_{\infty}}{\left(a^{2} \alpha^{2} q^{2 j}, a^{2} q^{j+1} ; q\right)_{\infty}}{ }_{2} \phi_{1}\left(\begin{array}{c|c}
\alpha^{2} / q a^{2}, q^{-n} / a^{2} & \\
\alpha^{2} q^{n} & q, a^{4} q^{n+j+1}
\end{array}\right) .
\end{aligned}
$$


According to (III.2) in [Ga:Ra] the above ${ }_{2} \phi_{1}$ can be transformed to

$$
\frac{\left(\alpha^{2} q^{j}, a^{2} \alpha^{2} q^{2 n}, a^{4} q^{2 j+1} ; q\right)_{\infty}}{\left(\alpha^{2} q^{n}, a^{2} \alpha^{2} q^{2 j}, a^{4} q^{n+j+1} ; q\right)_{\infty}}{ }_{2} \phi_{1}\left(\begin{array}{c|c}
q^{j-n}, q^{-n} / a^{2} & q, a^{2} \alpha^{2} q^{2 n} \\
a^{2} q^{j+1} &
\end{array}\right) .
$$

From this we find that Theorem 1 reduces to

$$
\begin{aligned}
& \left(\alpha e^{i \theta}, \alpha e^{-i \theta} ; q\right)_{n}=\sum_{j=0}^{n} \frac{(q ; q)_{n}(-\alpha)^{j} q^{j(j-1) / 2}}{(q ; q)_{n-j}\left(a^{2} ; q\right)_{j}} C_{j}\left(x ; a^{2} \mid q\right) \\
& \times{ }_{2} \phi_{1}\left(\begin{array}{c|c}
q^{j-n}, q^{-n} / a^{2} & q, a^{2} \alpha^{2} q^{2 n} \\
a^{2} q^{j+1} &
\end{array}\right.
\end{aligned}
$$

There are several choices for $\alpha^{2}$ for which the ${ }_{2} \phi_{1}$ series in (3.4) is summable. The choice of interest here is $\alpha=-i q^{(1-n) / 2}$, so that the Bailey-Daum formula [Ga:Ra, (II.9)] implies

$$
{ }_{2} \phi_{1}\left(\begin{array}{c|c}
q^{j-n}, q^{-n} / a^{2} & q,-a^{2} q^{n+1} \\
a^{2} q^{j+1} & \text { if } n-j \text { is odd, }
\end{array}\right.
$$

The appropriate choice of $c_{n}$ leads to

$$
\begin{aligned}
& \mathcal{E}_{q}(x ;-i, b / 2)=\sum_{j=0}^{\infty} \frac{q^{j^{2} / 4}(i b / 2)^{j}}{\left(a^{2} ; q\right)_{j}} C_{j}\left(x ; a^{2} \mid q\right) \\
& \times{ }_{2} \phi_{1}\left(\begin{array}{c|c}
-a^{2} q^{j+1},-a^{2} q^{j+2} & q^{2},-\frac{b^{2}}{4} \\
a^{4} q^{2 j+2} &
\end{array} .\right.
\end{aligned}
$$

Setting $a=q^{\nu / 2}$ in (3.5) and using (1.6), we see that (1.2) follows from (3.5).

By taking the limit $\nu \rightarrow \infty$ in (1.3) we find that

$$
\mathcal{E}_{q}(x ;-i, b)=\frac{1}{\left(-b^{2} ; q^{2}\right)_{\infty}} \sum_{n=0}^{\infty}(i b)^{n} q^{n^{2} / 4} \frac{H_{n}(x \mid q)}{(q ; q)_{n}},
$$

where

$$
H_{n}(x \mid q)=(q ; q)_{n} C_{n}(x ; 0 \mid q),
$$

are the continuous $q$-Hermite polynomials.

\section{4. $\mathcal{E}_{q}$ EXPANSiOnS IN SPECIAL Askey-Wilson polynomials}

In this section we evaluate $A_{n, j}$ using special balanced ${ }_{4} \phi_{3}$ 's. This naturally leads to expansions in special families of Askey-Wilson polynomials, see (4.1), (4.2), (4.3), (4.4). We also choose two specializations so that $A_{n, j}$ is transformable, yielding $\mathcal{E}_{q}$ expansions, including (1.3). At the end of the section we also prove the Al-SalamChihara polynomial result by generating functions.

Andrews' terminating $q$-analogue of the Watson's ${ }_{3} F_{2}$ sum is [Ga:Ra, (II.17)]

$$
{ }_{4} \phi_{3}\left(\begin{array}{l}
q^{-n}, A q^{n}, C,-C \\
\sqrt{A q},-\sqrt{A q}, C^{2}
\end{array} \mid q, q\right)=\left\{\begin{array}{l}
0 \quad \text { if } n \text { is odd, } \\
\frac{C^{n}\left(q, q A / C^{2} ; q^{2}\right)_{n / 2}}{\left(q A, q C^{2} ; q^{2}\right)_{n / 2}} \text { if } n \text { is even. }
\end{array}\right.
$$


This suggests that after replacing $n$ by $n+j$ in (2.5), we take

$$
\alpha=-i q^{(1-n) / 2}, \quad a=-b, \quad c=-d, \quad C= \pm i q^{(1-n-j) / 2} / a, \quad A=-c^{2} q^{j-n} .
$$

Therefore $A_{2 m+j+1, j}=0$, and

$$
\frac{A_{2 m+j, j}}{(q ; q)_{2 m+j}}=\frac{\left(a^{2} c^{2} / q ; q\right)_{j}(q ; q)_{j+2 m}\left(-a^{2} q^{j+1},-c^{2} q^{j+1} ; q^{2}\right)_{m}}{(q ; q)_{j}\left(a^{2} c^{2} / q ; q\right)_{2 j}\left(q^{2}, a^{2} c^{2} q^{2 j+1} ; q^{2}\right)_{m}} i^{j}(-1)^{m} q^{-m^{2}-m j}
$$

This leads to the expansion

$$
\begin{aligned}
\mathcal{E}_{q}(x ;-i, b)=\sum_{j=0}^{\infty} & \frac{\left(a^{2} c^{2} / q ; q\right)_{j}}{(q ; q)_{j}\left(a^{2} c^{2} / q ; q\right)_{j}} p_{j}(x ; a,-a, c,-c) \\
& \times(i b)^{j} q^{j^{2} / 4}{ }_{2} \phi_{1}\left(\begin{array}{c}
-a^{2} q^{j+1},-c^{2} q^{j+1} \\
a^{2} c^{2} q^{2 j+1}
\end{array} \mid q^{2},-b^{2}\right) .
\end{aligned}
$$

In [Is:Ma:Su] it was pointed out that

$$
\left(\frac{r}{2}\right)^{\nu} \frac{\left(q^{\nu+1},-r^{2} / 4 ; q\right)_{\infty}}{(q ; q)_{\infty}}{ }_{2} \phi_{1}\left(\begin{array}{c|c}
q^{(\nu+1) / 2} e^{i \theta}, q^{(\nu+1) / 2} e^{-i \theta} & q,-\frac{r^{2}}{4} \\
q^{\nu+1}
\end{array}\right)
$$

is a $q$-analogue of $J_{\nu}(x r), x=\cos \theta$. The ${ }_{2} \phi_{1}$ 's in (4.1) can be expressed in terms of the above $q$-Bessel function.

If we set $c=0$, then the ${ }_{2} \phi_{1}$ in $(4.1)$ becomes a ${ }_{1} \phi_{0}$, which can be summed by the $q$-binomial theorem [Ga:Ra, (II.3)]. We find

$$
\mathcal{E}_{q}(x ;-i, b)=\sum_{n=0}^{\infty} \frac{\left(-a^{2} ; q\right)_{n}\left(a^{2} b^{2} q^{n+1} ; q^{2}\right)_{\infty}}{(q ; q)_{n}\left(-b^{2} ; q^{2}\right)_{\infty}}\left(\frac{i b}{a}\right)^{n} q^{n^{2} / 4} r_{n}(x ; a,-a, 0,0) .
$$

Since (7.5.34) of [Ga:Ra] implies

$$
r_{n}\left(x ; q^{1 / 2},-q^{1 / 2}, 0,0\right)=\frac{q^{n / 2}}{(-q ; q)_{n}} H_{n}\left(x \mid q^{2}\right)
$$

we find that

$$
\mathcal{E}_{q}(x ;-i, b)=\sum_{n=0}^{\infty} \frac{\left(b^{2} q^{n+2} ; q^{2}\right)_{\infty}}{\left(-b^{2} ; q^{2}\right)_{\infty}(q ; q)_{n}}(i b)^{n} q^{n^{2} / 4} H_{n}\left(x \mid q^{2}\right),
$$

which may be compared with (3.6).

Another balanced ${ }_{4} \phi_{3}$ evaluation is [An, (4.3)]

$$
{ }_{4} \phi_{3}\left(\begin{array}{c}
q^{-2 n}, A^{2} q^{2 n}, B, B q \\
A q, A q^{2}, B^{2}
\end{array} \mid q^{2}, q^{2}\right)=B^{n} \frac{(A q / B,-q ; q)_{n}}{(A,-B ; q)_{n}} \frac{1-A}{1-A q^{2 n}} .
$$

So (2.2) is summable if $b=a q^{1 / 2}, d=c q^{1 / 2}$, and $\alpha^{2}=q^{1 / 2-n}$, or $\alpha^{2}=q^{3 / 2-n}$. 

is

After replacing $c$ by $-c$, the $\alpha=q^{(1-2 n) / 4}$ result with $c_{n}=q^{n(n+1) / 4}(i t / 2)^{n} /(q ; q)_{n}$

$$
\begin{aligned}
\mathcal{E}_{q}\left(x ; q^{-1 / 4}, i t q^{1 / 4} / 2\right)=\sum_{n=0}^{\infty} & \frac{\left(a^{2} c^{2} ; q\right)_{n}}{(q ; q)_{n}\left(a^{2} c^{2} ; q\right)_{2 n}} q^{n^{2} / 4}\left(-\frac{i t}{2}\right)^{n} \\
& \times p_{n}\left(x ; a, a q^{1 / 2},-c,-c q^{1 / 2}\right) \\
& \times{ }_{2} \phi_{1}\left(\begin{array}{c}
a q^{(2 n+1) / 4},-c q^{(2 n+1) / 4} \mid \\
a c q^{n+1 / 2}
\end{array} q^{1 / 2}, \frac{i t}{2}\right) .
\end{aligned}
$$

The choice of $\alpha=q^{(3-2 n) / 4}$ (thus expanding $\mathcal{E}_{q}\left(x ; q^{1 / 4}, i t q^{-1 / 4} / 2\right)$ ) gives the same expansion as (4.4). These two $\mathcal{E}_{q}$ functions are identical from [Su, (3.4)].

If, instead, we take $a=-i q^{(1-n) / 2}$, then the ${ }_{4} \phi_{3}$ series in (2.5) can no longer be summed. However the ${ }_{4} \phi_{3}$ series in $(2.5)$ can still be transformed to another ${ }_{4} \phi_{3}$ series in base $q^{1 / 2}$ by [Ga:Ra, (III.21)]. It turns out that the series over $n$ in (2.7), a double sum, can be simplified further by an interchange of the order of summation and finally reduced to a single sum. The result is equivalent to (1.3),

$$
\begin{gathered}
\mathcal{E}_{q}(x ;-i, t / 2)=\frac{\left(i t q^{1 / 2} / 2 ; q\right)_{\infty}}{(-i t / 2 ; q)_{\infty}} \sum_{n=0}^{\infty} \frac{\left(a^{2} c^{2} ; q\right)_{n} q^{n^{2} / 4}}{(q ; q)_{n}\left(a^{2} c^{2} ; q\right)_{2 n}}\left(-\frac{i t}{2}\right)^{n} \\
\times p_{n}\left(x ; a, a q^{1 / 2},-c,-c q^{1 / 2}\right) \\
\times{ }_{2} \phi_{1}\left(\begin{array}{c}
a q^{(2 n+1) / 4},-c q^{(2 n+1) / 4} \\
a c q^{n+1 / 2}
\end{array} \mid q^{1 / 2}, \frac{i t}{2}\right) .
\end{gathered}
$$

The right sides of (4.4) and (4.5) are identical. This follows from Suslov's addition theorem for the $\mathcal{E}_{q}$ functions; see [Su, Theorem 3.1].

We have another example when a ${ }_{4} \phi_{3}$ transformation, not a summation, leads to an $\mathcal{E}_{q}$ expansion. The Al-Salam-Chihara polynomials are defined by

$$
p_{n}(x ; a, b):=r_{n}(x ; a, b, 0,0) .
$$

We have (see also [Fl:Le:Vi2], (3.17))

$$
\begin{aligned}
\mathcal{E}_{q}(x ;-i, b)=\sum_{n=0}^{\infty} & \frac{\left(-\gamma^{2} b^{2} q^{n} ; q^{2}\right)_{\infty}}{\left(-b^{2} ; q^{2}\right)_{\infty}}(i b)^{n} q^{n^{2} / 4} p_{n}\left(x ; \gamma e^{i \phi}, \gamma e^{-i \phi}\right) \\
& \times \gamma^{-n} e^{-i n \phi} \frac{\left(\gamma^{2} ; q\right)_{n}}{(q ; q)_{n}} \mathcal{E}_{q}\left(\cos \phi ;-i, \gamma b q^{n / 2}\right) .
\end{aligned}
$$

To prove (4.6) we find the coefficient of $b^{n} p_{j}\left(x ; \gamma e^{-i \phi}, \gamma e^{i \phi}\right)$ on both sides. The left-side coefficient (by (2.7) and (2.5)) is a ${ }_{3} \phi_{2}$ on base $q$ with one denominator parameter equal to 0 . The right-side coefficient (by the $q$-binomial theorem) gives a ${ }_{3} \phi_{2}$ on base $q^{2}$. These are equal using [Ga:Ra, (3.10.13), (3.2.2)].

The preceding sketch of the proof of (4.6) is not transparent, so we also prove (4.6) from earlier results in this paper.

The Al-Salam-Chihara polynomials have the generating function [As:Is2]

$$
\sum_{n=0}^{\infty} \frac{\left(t_{1} t_{2} ; q\right)_{n}}{(q ; q)_{n}}\left(t / t_{1}\right)^{n} p_{n}\left(\cos \theta, t_{1}, t_{2}\right)=\frac{\left(t t_{1}, t t_{2} ; q\right)_{\infty}}{\left(t e^{i \theta}, t e^{-i \theta} ; q\right)_{\infty}} .
$$


The continuous $q$-Hermite polynomials, see (3.10), have the generating function [As:Is]

$$
\sum_{n=0}^{\infty} H_{n}(\cos \theta \mid q) t^{n} /(q ; q)_{n}=1 /\left(t e^{i \theta}, t e^{-i \theta} ; q\right)_{\infty} .
$$

Evidently the above two generating functions imply

$$
\frac{H_{n}(x \mid q)}{(q ; q)_{n}}=\sum_{j=0}^{n} \frac{H_{j}(\cos \phi \mid q)}{(q ; q)_{j}} p_{n-j}\left(x ; \gamma e^{i \phi}, \gamma e^{-i \phi}\right) \gamma^{2 j-n} e^{-i(n-j) \phi} \frac{\left(\gamma^{2} ; q\right)_{n-j}}{(q ; q)_{n-j}}
$$

We then combine (4.7) and (3.6) to obtain

$$
\begin{aligned}
\mathcal{E}_{q}(x ;-i, b)= & \frac{1}{\left(-b^{2} ; q^{2}\right)_{\infty}} \sum_{n=0}^{\infty}(i b)^{n} q^{n^{2} / 4} p_{n}\left(x ; \gamma e^{i \phi}, \gamma e^{-i \phi}\right) \gamma^{-n} e^{-i n \phi} \frac{\left(\gamma^{2} ; q\right)_{n}}{(q ; q)_{n}} \\
& \left.\times \sum_{j=0}^{\infty} \frac{H_{j}(\cos \phi \mid q)}{(q ; q)_{j}}\right) \gamma^{j} q^{j^{2} / 4}\left(i b q^{n / 2}\right)^{j}
\end{aligned}
$$

The $j$ sum can be expressed in terms of $\mathcal{E}_{q}$ by (3.6) to obtain (4.6).

It is not hard to see that choosing $\phi=\pi / 2, \gamma=-i \sqrt{q}$ in (4.6) gives (4.3).

\section{Connection COefficients for Askey-Wilson polynomials}

From (2.2) and (2.7) we have

$$
r_{m}(x ; \alpha, \beta, \gamma, \delta)=\sum_{j=0}^{m} B_{m, j} r_{j}(x ; a, b, c, d),
$$

where

$$
\begin{aligned}
B_{m, j}= & \frac{\left(a b, a c, a d, q^{-m}, \alpha \beta \gamma \delta q^{m-1}, a b c d / q ; q\right)_{j}}{(\alpha \beta, \alpha \gamma, q, a b c d / q ; q)_{j}} \\
& \times\left(-\frac{\alpha}{a}\right)^{j(j+1) / 2} \sum_{n=0}^{m-j} \frac{\left(q^{j-m}, \alpha \beta \gamma \delta q^{m+j-1}, \alpha d q^{j}, \alpha / d ; q\right)_{n}}{\left(q, \alpha \beta q^{j}, \alpha \gamma q^{j}, \alpha \delta q^{j} ; q\right)_{n}} q^{n} \\
& \times{ }_{4} \phi_{3}\left(\begin{array}{c}
q^{-n}, a d q^{j}, b d q^{j}, c d q^{j} \\
d q^{1-n} / \alpha, \alpha d q^{j}, a b c d q^{2 j}
\end{array} \mid q, q\right) .
\end{aligned}
$$

We shall assume that

$$
\max (|a|,|b|,|c|,|d|)<1
$$

and that $\alpha$ and $a$ are of the same sign. Askey and Wilson [As:Wi] have shown that the connection coefficients are positive when

$$
0<\alpha<a<1, \quad b=\beta, \quad c=\gamma, \quad d=\delta .
$$

This can be iterated using the symmetry of $p_{n}(x ; a, b, c, d)$ in its parameters. Thus we have proven that the $B_{m, j}$ of (5.1) is nonnegative for

$$
0<\alpha<a<1, \quad 0<\beta<b<1, \quad 0<\gamma<c<1, \quad 0<\delta<d<1 .
$$




\section{MATRIX INVERSION}

In this section we prove Theorem 1 from an explicit matrix inversion.

Let

$$
\phi_{n}(x ; a)=\left(a e^{i \theta}, a e^{-i \theta} ; q\right)_{n}, \quad x=\cos \theta .
$$

It follows from [Ga:Ra, (II.12)] that

$$
\phi_{n}(x, \alpha)=\sum_{k=0}^{n} C_{n k} \phi_{k}(x, a),
$$

where

$$
C_{n k}=\frac{q^{k}\left(q^{-n} ; q\right)_{k}(a \alpha, \alpha / a ; q)_{n}}{\left(q, a \alpha, a q^{1-n} / \alpha ; q\right)_{k}} .
$$

To establish Theorem 1, it remains to expand $\phi_{k}(x, a)$ in terms of the AskeyWilson polynomials $p_{j}(x ; a, b, c, d)$ by inverting

$$
p_{n}(x ; a, b, c, d)=\sum_{j=0}^{n} D_{n j}(t) c_{j} \phi_{j}(x, a),
$$

where

$$
D_{n j}(t)=\left(q^{-n}, t q^{n} ; q\right)_{j}, \quad t=a b c d / q, \quad 1 / c_{j}=q^{-j}(q, a b, a c, a d ; q)_{j} .
$$

It is known (see for example (3.6.19) and (3.6.20) in [Ga:Ra]) that

$$
D_{j k}^{-1}(t)=\frac{t^{-k} q^{j-k^{2}}}{\left(q, q^{1-2 k} / t ; q\right)_{k}\left(q, t q^{1+2 k} ; q\right)_{j-k}} .
$$

Thus Theorem 1 follows from the matrix inversion (6.1). Since there is bibasic version of (6.1), there is also a bibasic version of Theorem 1 .

We can also find the inverse of the lower triangular matrix $A_{n, j}$ of Theorem 1 by expanding the polynomials $p_{j}(x ; a, b, c, d)$ in terms of $\phi_{l}(x ; \alpha)$. Using $(6.1)$ we find

$$
\begin{aligned}
A_{j, l}^{-1}=q^{l} & a^{l-j} \alpha^{-l}\left(a b q^{l}, a c q^{l}, a d q^{l} ; q\right)_{j-l} \frac{\left(a b c d q^{j-1}, q^{-j} ; q\right)_{l}}{(q ; q)_{l}} \\
& \times{ }_{4} \phi_{3}\left(\begin{array}{c}
q^{l-j}, a b c d q^{j+l-1}, a / \alpha, a \alpha q^{l} \\
a b q^{l}, a c q^{l}, a d q^{l}
\end{array} \mid q, q\right)
\end{aligned}
$$

which gives a discrete orthogonality relation for a ${ }_{4} \phi_{3}$.

\section{REFERENCES}

[Al:Ch] W. A. Al-Salam and T. S. Chihara, Convolutions of orthogonal polynomials, SIAM J. Math. Anal. 7 (1976), 16-28. MR 53:3381

[An] G. E. Andrews, Connection coefficient problems and partitions, Proc. Symp. Pure Math 34 (1979), 1-24. MR 80c:33004

[As:Is] R. A. Askey and M. E. H. Ismail, A generalization of ultraspherical polynomials, Studies in Pure Mathematics (P. Erdös, ed.), Birkhauser, Basel, 1983, pp. 55-78. MR 87a:33015

[As:Is2] R. A. Askey and M. E. H. Ismail, Recurrence relations, continued fractions and orthogonal polynomials, Memoirs Amer. Math. Soc. 49 (1984). MR 85g:33008

[As:Wi] R. A. Askey and J. A. Wilson, Some basic hypergeometric orthogonal polynomials that generalize Jacobi polynomials, Memoirs Amer. Math. Soc. 54 (1985). MR 87a:05023

[At:Su] N. M. Atakishiev and S. K. Suslov, Difference hypergeometric functions, Progress in Approximation Theory, An International Perspective (A. A. Gonchar and E. B. Saff, ed.), Springer-Verlag, New York, 1992, pp. 1-35. MR 94k:33039 
[Fi:Is] J. Fields and M. E. H. Ismail, Polynomial expansions, Mathematics of Computation 29 (1975), 894-902. MR 51:8680

[Fl:Vi] R. Floreanini and L. Vinet, A model for the continuous q-ultraspherical polynomials, J. Math. Phys. 36 (1995), 3800-3813. MR 96d:33012

[Fl:Le:Vi] R. Floreanini, J. LeTourneux and L. Vinet, More on the q-oscillator algebra and qorthogonal polynomials, Journal of Physics A 28 (1995), L287-L293. MR 96e:33043

[Fl:Le:Vi2] R. Floreanini, J. LeTourneux and L. Vinet, Symmetry techniques for the Al-SalamChihara polynomials, J. Phys. A 30 (1997), 3107-3114. MR 98k:33036

[Ga:Ra] G. Gasper and M. Rahman, Basic Hypergeometric Series, Cambridge University Press, Cambridge, 1990. MR 91d:33034

[Ge:St] I. Gessel and D. Stanton, Applications of q-Lagrange inversion to basic hypergeometric series, Trans. Amer. Math. Soc. 277 (1983), 173-201. MR 84f:33009

[Is] M. E. H. Ismail, The zeros of basic Bessel functions the functions $J_{\nu+a x}(x)$ and the associated orthogonal polynomials, J. Math. Anal. Appl. 86 (1982), 1-19. MR 83c: 33010

[Is:Ma:Su] M. E. H. Ismail, D. Masson and S. Suslov, The q-Bessel functions on q-quadratic grid (to appear).

[Is:Ra:Zh] M. E. H. Ismail, M. Rahman and R. Zhang, Diagonalization of certain integral operators II, J. Comp. Appl. Math. 68 (1996), 163-196. MR 98d:33011

[Is:Zh] M. E. H. Ismail and R. Zhang, Diagonalization of certain integral operators, Advances in Math. 109 (1994), 1-33. MR 96d:39005

[Lu] Y. Luke, The Special Functions and Their Approximations, volume 2, Academic Press, New York, 1969. MR 40:2909

[Ra] M. Rahman, An integral representation and some transformation properties of $q$ Bessel functions, J. Math. Anal. Appl. 125 (1987), 58-71. MR 88h:33020

[Su] S. K. Suslov, Addition theorems for some q-exponential and trigonometric functions, Methods and Applications of Analysis 4 (1997), 11-32. CMP 97:14

Department of Mathematics, University of South Florida, Tampa, Florida 33620-5700

E-mail address: ismail@math.nsf.edu

Department of Mathematics, Carleton University, Ottawa, Ontario, Canada K1S $5 \mathrm{~B} 6$

E-mail address: mrahman@math.carleton.ca

School of Mathematics, University of Minnesota, Minneapolis, Minnesota 55455

E-mail address: stanton@math.umn.edu 